\title{
Change of Cochlear Micromechanics due to Different Types of Hearing Loss
}

\author{
Guangjian Ni and Stephen Elliott, Member, IEEE
}

\begin{abstract}
The reasons for hearing loss are complex and currently the mechanics are not entirely clear. Outer hair cell (OHC) loss is believed to play an important role. Experimental observations shown that damage on OHCs due to ototoxic acid starts from the outermost row to the innermost row, whereas, loss of $\mathrm{OHCs}$ due to intense noise exposure occurs from the innermost row to the outermost row. Inspired by these experiments, this study employs the finite element method to develop a detailed model of a slice of the human cochlea including cochlear fine structures. OHC motility is implemented by applying forces at the two ends of the OHCs in response to stereocilia deflection, which are believed to be a key process in cochlear amplification. In this way, the effects of a loss of OHCs due to either intense noise exposure or ototoxic acid can be studied by suppressing forces on individual OHCs. Change of cochlear mechanical amplification and vibration patterns inside the organ of Corti due to different hearing loss mechanisms can thus be predicted.
\end{abstract}

\section{INTRODUCTION}

$\mathrm{O}$ UTER hair cells (OHCs) are well known to play an essential role in the cochlear amplification. When the basilar membrane (BM) is excited by the fluid pressure difference between the cochlear fluid chambers, the stereocilia of the OHCs are deflected by the shearing motion between the reticular lamina (RL) and the tectorial membrane (TM), which then opens transduction channels and changes OHCs intracellular potential. The length of OHCs will change accordingly and then generate forces acting on the BM via the Deiters' cells (DC) to amplify BM motion.

Outer hair cell loss is believed to play an essential role in hearing loss caused by various reasons. Experimental observations shown that damage on OHCs due to ototoxic acid starts from the outermost row to the innermost row, whereas, loss of OHCs due to intense noise exposure occurs from the innermost row to the outermost row $[1,2]$. In the basal turn, $\mathrm{OHC}$ loss was observed in almost all of the noise-exposed rats, but inner hair cells (IHC) loss was still not observed until $50 \mathrm{~dB}$ CAP threshold elevation [1]. Inner hair cells are also relatively insensitive to styrene exposure, since the maximal cochlear action potential amplitude remains

Manuscript received September 19, 2015. This research is supported by EU project SIFEM (Grant FP7-600933) on the multi-scale modelling and 3D visualization for the inner-ear.

Guangjian $\mathrm{Ni}$ is with Institute of Sound and Vibration Research, University of Southampton, SO17 1BJ UK (e-mail: G.Ni@soton.ac.uk).

Stephen Elliott is with Institute of Sound and Vibration Research, University of Southampton, SO17 1BJ UK (e-mail: S.J.Elliott@soton.ac.uk). unchanged even when the OHCs, the related DC and some of the outer pillar cells are destroyed [2].

Models of the cochlea have been used extensively to reproduce the results from a large variety of experiments and to provide hypothesis to explain phenomena remaining unclear. This paper presents a finite element 2D model of the human cochlea containing detailed organ of Corti (OC) and studies effects of the $\mathrm{OHC}$ loss on the cochlear amplification. The model was verified in two aspects: 1) its resonance frequency meets the characteristic frequency $(\mathrm{CF})$ of the place where its geometry was extracted and 2) its vibration pattern under passive acoustic stimuli agrees reasonable well with experiment.

\section{MiCROMECHANICAL MODEL OF THE HUMAN COCHLEA}

Modelling the cochlea is a multi-scale problem, since the bone of the partition has dimensions on the millimeter $\left(10^{-3} \mathrm{~m}\right)$ scale, the hair cells and supporting cells on the micrometer $\left(10^{-6} \mathrm{~m}\right)$ scale, and the tip links connecting stereocilia having thickness on the nanometer $\left(10^{-9} \mathrm{~m}\right)$ scale [3]. The micromechanical model is built based on geometries from the human cochlea. Different types of cells and fluid spaces are represented by closed areas. In the model, as shown in Fig. 1, elastic components are modelled with Ansys (V15.0) plane42 element, which is defined by four nodes having two degrees of freedom, translations in the vertical and horizontal directions, at each node. The viscous fluid is modelled with a secondary fluid element, fluid79, which is a modification of the plane element and is defined also by four nodes having two degrees of freedom, particle displacement in the nodal $x$ and $y$ directions, at each node.

At the position where the geometry is obtained, the basilar membrane is connected with spiral lamina on its left hand side and with the spiral ligament on the other side. The real boundary condition for the BM is complicated and depends on neighboring tissues and fibers. Homer et al. [4] show that a simple beam model of the BM is capable of simulating the BM deflection profile observed by Cooper [5] at all frequencies. For their model, the BM is simply-supported at the left end (at the spiral lamina) and clamped at the other end (at the spiral ligament). Steele and Puria [6] applied clamped condition for both ends in their 2D finite element model of the $\mathrm{OC}$ and emphasis that the clamping on the right side of the BM is important for the guinea pig. Ni and Elliott [7] suggested that the fluid coupling and the coupled response are not critically dependent on the BM boundary conditions and the fluid 
coupling in cochlear models can be reasonably well estimated by assuming a single, fixed, radial profile. In this way, we assume that the BM of the present model is clamped at its two ends, which is consistent with Steele and Puria [6] model.

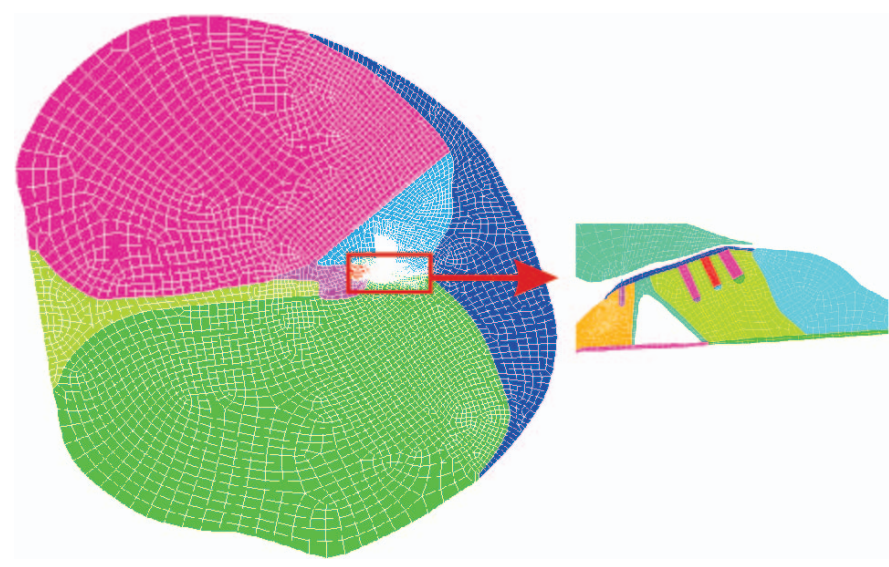

Fig. 1. FE model of the cochlea corresponding to the position $12 \mathrm{~mm}$ away from the base.

TABLE I

MATERIAL PROPERTIES

\begin{tabular}{cc}
\hline \hline Quantity & Young's Modulus [Pa] \\
\hline Basilar membrane & $9.83 \times 10^{8}$ \\
Tectorial membrane & $1 \times 10^{3}$ \\
Hensen's cells & $9.83 \times 10^{3}$ \\
Inner hair cell & $1 \times 10^{2}$ \\
Outer hair cells & $1 \times 10^{5}$ \\
Deiter cells & $5 \times 10^{3}$ \\
Spiral ligament & $1 \times 10^{10}$ \\
Spiral lamina & $1 \times 10^{10}$ \\
Modiulus & $1 \times 10^{9}$ \\
Pillar cells & $1 \times 10^{6}$ \\
Reticular lamina & $1 \times 10^{9}$ \\
Stereocilia & $1 \times 10^{6}$ \\
\hline \hline
\end{tabular}

The material properties of the cochlear fluid and elastic components of the models are tuned based the frequency-position relation. The difference of elastic modulus between adjacent components in the cochlea may vary by orders of magnitude. For example, the TM has a modulus around $1 \mathrm{kPa}$ in the apical turn [8], but the bone of the partition and walls has a modulus around $20 \mathrm{GPa}$ [9]. Generally, with knowledge of the protein fibers, an estimate can be made for the effective stretching and bending stiffness of a particular component. However, uncertainty remains, for example, in the volume fraction of protein in a particular component and in the stiffness of ground substance. Some important material properties of the model are list in Table I. Poisson's ratio of components whose Young's modulus are less than $1 \times 10^{6} \mathrm{~Pa}$ is assumed to be 0.45 to 0.49 since these cells are assumed to be nearly incompressible. Poisson's ratio of the other components with greater Young's modulus is assigned to be 0.3 . The density for all components is set to that of water, $1 \times 10^{3} \mathrm{~kg} \cdot \mathrm{m}^{-3}$. The dynamic viscosity of the cochlear fluid is set to $0.894 \times 10^{-3} \mathrm{~Pa} \cdot \mathrm{s}$ and the bulk modulus is equal to that of water $2.25 \mathrm{GPa}[10]$.

\section{MODEL VERIFICATION}

It is fundamental for the model to be able to reflect the correct frequency-position map in the human auditory system. In the model, this is achieved by tuning material parameters that define the stiffness and mass of the cochlear fluid and elastic components. An analytic description of the frequency-position map for the human ear was estimated by Greenwood [11] as

$C F=A\left(10^{\alpha L-x}-k\right)$,

where $A=165.4$ to yield frequency in $\mathrm{Hz}, x$ is the distance from the base, $L$ is the length of the cochlea, $a=0.06$ as $x$ is expressed in millimeter. The constant $k$ determines the lowest frequency limit of the mapping, with $k=0.88$ giving a value of $20 \mathrm{~Hz}$. We therefore tune of the model material properties based on Greenwood's function to gain physiological reality.

Figure 2 shows the BM displacement amplitude and phase as a function of driving frequency when OHCs motility is not presented. It can be seen that the BM displacement gradually increases until reaches a peak at about $3.2 \mathrm{kHz}$ and then decays away quickly. This resonance frequency is close to the characteristic frequency of the position to which the model corresponds.
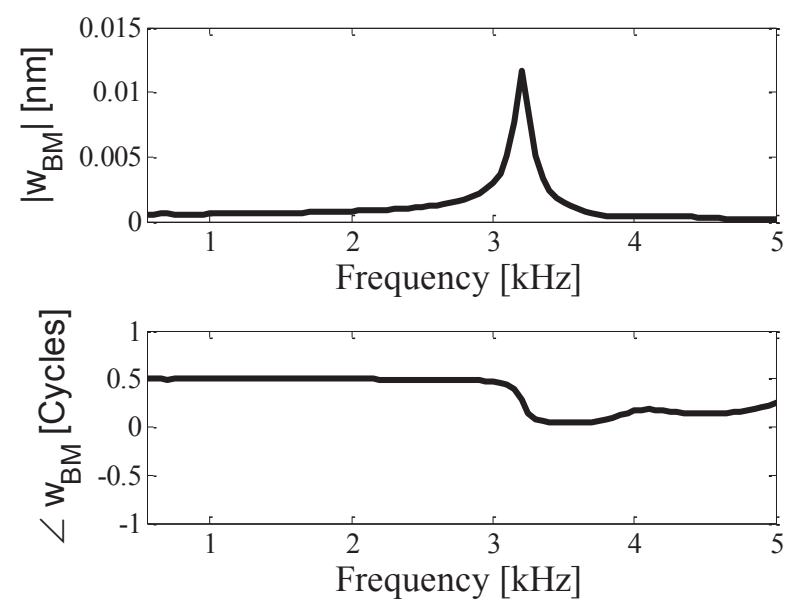

Fig. 2. The predicted BM displacement in response to a passive acoustic excitation of $20 \mathrm{~dB}$ SPL.

Figure 3 shows the motion of the organ of Corti at $3.2 \mathrm{kHz}$ when a passive acoustic pressure is applied below and above the $\mathrm{BM}$ simulating the condition that the passive $\mathrm{BM}$ is driven by a fluid pressure difference at the characteristic frequency. The whole organ of Corti moves in phase and the BM displacement increases linearly from the feet of the inner hair cell to the feet of the outer pillar cells and the amplitude is maximized close to the second row of the OHCs. This is 
consistent with the observations of Fridberger and Boutet de Monvel [13], in which motions within the organ of Corti are measured at a high level excitation and a drug is used to block the OHCs electromotility. A clear shearing motion of the stereocilia between the reticular lamina and tectorial membrane can also be seen to move outwards, which would cause depolarization (contraction) of the $\mathrm{OHCs}$, if $\mathrm{OHCs}$ electromotility was included.

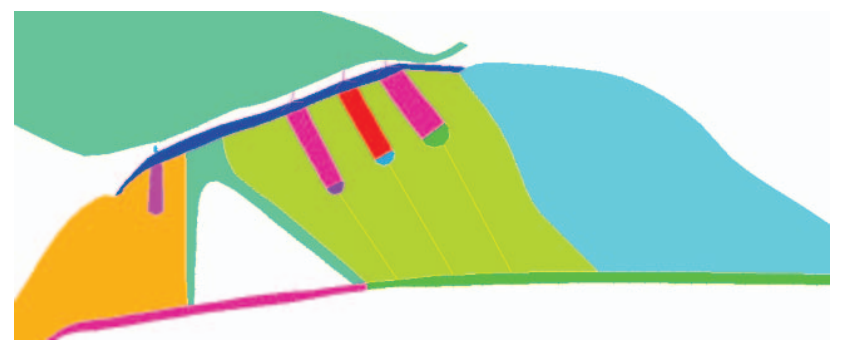

Fig. 3. Vibration pattern of the organ of Corti at $3.2 \mathrm{kHz}$ when the model is driven by a passive acoustic pressure of $20 \mathrm{~dB}$ SPL.

\section{OUTER HAIR CELLS EXCITATION}

In order to model the effect of OHCs electromotility, forces with opposite sign are applied in the model at the two ends of the OHCs, along their axial direction, to simulate depolarization of membrane potential and contraction of the OHCs. The magnitude of OHCs force is assumed to depend on the shearing motion, $\delta$, between the reticular lamina and the tectorial membrane as [12][13]

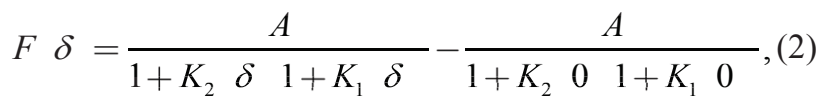

where

$$
K_{1,2}=e^{a_{1,2} \delta_{1,2}-\delta} \text {, }
$$

and $A$ is the maximum force generated by the OHC motility and can be estimated to be $155 \mathrm{nN}$ to achieve a $30 \mathrm{~dB}$ amplification of the BM at $16 \mathrm{kHz}$ with $20 \mathrm{~dB}$ SPL acoustic stimulus, $K_{1,1}$ are the equilibrium constants of the two transitions, $\delta_{1,2}$ are the shearing displacement at which the set points of transition between states are found, estimated to be $0.092 \mathrm{~nm}^{-1}$ and $0.038 \mathrm{~nm}^{-1}$, and $a_{1,2}$ are the shearing displacement sensitivities of the transitions, estimated to be $8.2 \mathrm{~nm}$ and $49 \mathrm{~nm}$ [12]. Although these fitting parameters are estimated at $16 \mathrm{kHz}$, which is different from the $\mathrm{CF}$ of the model, $3.2 \mathrm{kHz}$, presented here, we assume that $\mathrm{OHC}$ physiological features remain the same.

It can be seen from Fig. 4 that the BM displacement profile is not sensitive to the absence of OHCs motility. The amplitude is maximized, not surprisingly, when all three OHCs are active. In the case of exposing to intense noise, which leads to OHCs functional loss from the innermost row, the reductions of the mechanical amplification are $1 \%$ (first row removed), 42\% (first and second rows removed) and $100 \%$ (all three rows removed). In the case of ototoxic acid, which leads to OHCs function loss from the outermost row, the reductions are 58\% (third row removed), 86\% (second and third rows removed) and 100\% (all three rows removed). The level of amplification reduction from this human cochlear model is smaller compared with those from a gerbil cochlear model [13], but the conclusion that the third row of the $\mathrm{OHC}$ plays the most important role in the mechanical amplification is the same with the gerbil model.

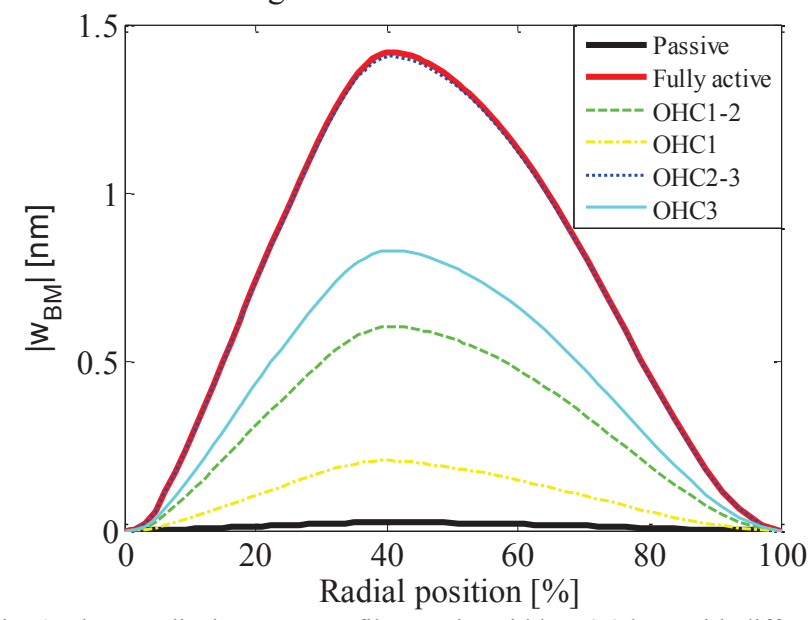

Fig. 4. The BM displacement profile over its width at $3.2 \mathrm{kHz}$ with different types $\mathrm{OHC}$ loss, together with the conditions of fully active (all $\mathrm{OHCs}$ are functional) and fully passive (all $\mathrm{OHCs}$ are not functional). OHC1-2 means the first and second row of $\mathrm{OHCs}$ are functional, $\mathrm{OHC} 1$ means only the first row of $\mathrm{OHC}$ is functional, $\mathrm{OHC} 2-3$ means the second and the third row of $\mathrm{OHC}$ are functional and $\mathrm{OHC} 3$ means only the third row of $\mathrm{OHC}$ is functional.

\section{CONCLUSION}

A micromechanical model of the human cochlea is developed using the finite element method to study effects of $\mathrm{OHCs}$ loss to the BM mechanical amplification. The BM displacement profile is found to be insensitive to the OHCs loss. The third row of the OHCs is seen to provide most contributions to the BM mechanical amplification. Although the numerical predictions cannot fully reproduce observations from experiments [1][2], the numerical model is able to show a similar tendency. Most importantly, the human cochlear models enable us to study possible effects of OHCs loss, which cannot be done experimentally.

Acknowledgments

This research is supported by EU project SIFEM (Grant FP7-600933) on the multi-scale modelling and 3D visualization for the inner-ear.

\section{REFERENCES}

[1]G. D. Chen and L. D. Fechter, "The relationship between noise-induced hearing loss and hair cell loss in rats," Hear Res, vol. 177, pp. 81-90, Mar 2003.

[2]G. D. Chen, C. Tanaka, and D. Henderson, "Relation between outer hair cell loss and hearing loss in rats exposed to styrene," Hear Res, vol. 243, pp. 28-34, Sep 2008.

[3]M. LeMasurier and P. G. Gillespie, "Hair-Cell Mechanotransduction and Cochlear Amplification," Neuron, vol. 48, pp. 403-415, 11/3/ 2005.

[4]M. Homer, A. Champneys, G. Hunt, and N. Cooper, "Mathematical modeling of the radial profile of basilar membrane vibrations in the inner ear," The Journal of the Acoustical Society of America, vol. 116, pp. 1025-1034, 2004. 
[5]N. P. Cooper, "Radial variation in the vibrations of the cochlear partition," presented at the Recent Developments in Auditory Mechanics, Singapore, 1999.

[6]C. R. Steele and S. Puria, "Force on inner hair cell cilia," International Journal of Solids and Structures, vol. 42, pp. 5887-5904, 10// 2005.

[7]G. Ni and S. J. Elliott, "Effect of basilar membrane radial velocity profile on fluid coupling in the cochlea," J. Acoust. Soc. Am. , vol. 133, pp. EL181-EL187, 2013.

[8]H. Cai and R. Chadwick, "Radial structure of traveling waves in the inner ear," SIAM Journal on Applied Mathematics, vol. 63, pp. 1105-1120, 2003. [9]C. R. Steele, G. J. Baker, J. A. Tolomeo, and D. E. Zetes-Tolometo, "Cochlear Mechanics," in The Biomedical Engineering Handbook, J. D. Bronzino, Ed., 2 ed Boca Raton: CRC Press, 2000, p. 3024.

[10] F. Böhnke and W. Arnold, "3D-Finite Element Model of the Human Cochlea Including Fluid-Structure Couplings," ORL, vol. 61, pp. 305-310, 1999.

[11] D. D. Greenwood, "Critical Bandwidth and the Frequency Coordinates of the Basilar Membrane," The Journal of the Acoustical Society of America, vol. 33, pp. 1344-1356, 1961.

[12] G. S. Geleoc, G. W. Lennan, G. P. Richardson, and C. J. Kros, "A quantitative comparison of mechanoelectrical transduction in vestibular and auditory hair cells of neonatal mice," Proc Biol Sci, vol. 264, pp. 611-21, Apr 221997.

[13] M. Murakoshi, S. Suzuki, and H. Wada, "Murakoshi: Coordinated Movement of the Three Rows of Outer Hair Cells is Essenti," presented at the 12th Mechanics of Hearing, Cape Sounio, Greece, 2014. 